\title{
Analysis of the Prevalence of the Preferred Methods of Contraception During the COVID 19 Lockdown in Uganda: A Multinomial Logistic Regression Study.
}

Julius Sseninde ( $\sim$ juliusprh@gmail.com )

Makerere University College of Business and Management Sciences https://orcid.org/0000-0002-95550560

\section{Allen Kabagenyi}

Makerere University College of Business and Management Sciences

\section{Betty Kyadondo}

National Population Council

\section{Evelyne Nyachwo}

Makerere University College of Health Sciences

\section{Agnes Kiragga}

Makerere University Infectious Diseases Institute

\section{Ronald Wasswa}

Makerere University College of Business and Management Sciences

\section{Research Article}

Keywords: COVID-19, lockdown, contraception, Uganda

Posted Date: June 17th, 2021

DOl: https://doi.org/10.21203/rs.3.rs-635015/v1

License: (c) (i) This work is licensed under a Creative Commons Attribution 4.0 International License.

Read Full License 


\title{
Analysis of the Prevalence of the Preferred Methods of Contraception During the COVID 19 Lockdown in Uganda: A Multinomial Logistic Regression Study.
}

Julius Sseninde' Allen Kabagenyi', Betty Kyaddondo', and Agnes Kirraga ${ }^{4}$, Evelyne Nyacho Ronald Wasswa ${ }^{1}$ (1) Population Studies, Makerere University, Kampala, Uganda, (2) National Population Council, Kampala, Uganda, (3) Community health, Makerere University School of Public Health, Kampala, Uganda, (4) Institute of Infectious diseases, Research Unit, Makerere University, Kampala, Uganda

\begin{abstract}
Background

In 2020 limitations to family planning information, access and utilization were exacerbated by the COVID-19 pandemic and lockdown, globally incapacitating various channels to family planning information, access and, utilization. This study sought to analyze the prevalence of the preferred methods of contraception during the COVID 19 lockdown in Uganda using multinomial logistic regression.
\end{abstract}

\section{Method}

Data were analyzed using univariate, bivariate, logistic, and multinomial logistic regression models from the post-intervention telehealth cross-sectional survey.

\section{Results}

Out of the 244 women surveyed, more than three quarters (80\%) used their preferred methods of family planning, while almost half of the participants (46\%) used the Short-Acting Methods as a preferred method of Family planning. The logistic regression showed that older-aged participants, fear of security personnel, and fear that my spouse will know that the partner is using family planning as their biggest challenge plus participants who agreed that they won't continue using family planning after the lockdown were less likely to use the preferred method of family planning. However, participants whose biggest challenge to accessing family planning information, and utilization was lack of money, those who received an extremely adequate number of messages during the intervention, and those who were already using family planning before the intervention were more likely to use their preferred method of family planning.

From the multinomial model, participants whose biggest challenges in accessing family planning services during the lockdown were fear of unwanted pregnancy, and fear if implant expires in her body were relatively more likely to use Long-term Acting Reversible Contraceptives than Short-Acting Contraceptives as their preferred method of Family Planning. While participants who were relatively less likely to use LARC compared to SAC were those already using family planning before the start of the intervention.

\section{Conclusions}

We recommend prioritizing family planning information, access and utilization as one of the essential services during the lockdown, with more emphasis on an intervention that addresses age-specific needs and partner privacy issues. Addressing the use of LARC among women who are already using contraceptives is equally paramount in cases of lockdown and health facility closures 


\section{Background}

In 2020 limitations to family planning information, access and utilization was exacerbated by COVID-19; a new disease, caused by a novel coronavirus. The sudden lockdown during the pandemic incapacitated various channels of access to family planning (Riley et al., 2020; Roberton et al., 2020). Globally unmet need for Family planning has always been a major public health concern over decades leading to mistimed and unwanted pregnancies and induced abortions. This is negatively associated with the social, economic and health issues of women and their families (Ahinkorah et al., 2020). Evidence suggests that the COVID 19 pandemic has disrupted access to all family planning information and services. Clinical staff are now more than ever occupied with the COVID-19 response, health facilities are limiting access to other public health services, women are refraining from accessing health facilities for fear of corona virus while supply chain has been distorted leading to shortages of FP products(Riley et al., 2020). Therefore, identifying unique channels through which sexually active clients can easily access contraceptive methods during the COVID 19 lockdown is essential for the success of Family Planning programs

Before the advent of COVID 19, 190 million women of reproductive age worldwide, who wanted to avoid pregnancy did not use any method of contraception (Tewabe et al., 2020). Currently, with all the available modern contraceptive methods to select from, mistimed and unwanted pregnancies, induced abortions, and higher fertility rates continue to stagnate. Adverse events of limitations to family planning information, access, and utilization put a lot of weight on the social-economic sectors of a country. Such effects negatively impact maternal and child outcomes leading to underutilization of prenatal and antenatal care. and infant mortality, common in unwanted pregnancies with potentially major long-term implications (Roberton et al., 2020). Evidence from public health studies supports an association between maternal mortality, unsafe abortion, and higher fertility rates with low access to Family Planning services (Ahmed et al., 2012; Cleland et al., 2006; Ganatra \& Faundes, 2016). There is accumulating evidence that women in Sub-Saharan (low-income countries) with unmet need for family planning, are availed with contraceptive methods, not of their choice (Do \& Kurimoto, 2012). There is more evidence that women in low-income countries are convinced by the health facility personnel to take what is available other than the most preferred methods of Family planning (Bitzer et al., 2012; Chigbu et al., 2010; Do \& Kurimoto, 2012). These studies have attributed women to negligence in adhering to family planning uptake.

The availability of various safe modern contraceptive methods at the health facility rarely guarantees the use of family planning. While counseling is pivotal in ensuring uptake of family planning, several studies are indicative of the influence of the patient-provider role, with a bias towards some methods which result in contraceptive discontinuation, or method switching. Such misinformation escalates the burden of using a method that is not preferred ( Bitzer et al., 2012; Do \& Kurimoto, 2012). Already this negative situation is worsened by the COVID 19 lockdown where a patient gets stack with an implant, a copper IUD plus all the side effects brought about by contraceptives like breakthrough bleeding, nausea, breast tenderness, headaches, weight gain, and mood swings ( Dasgupta et al., 2020; Sharma et al., 2020). With the reluctance of the providers in providing the preferred method of family planning other than what is available, resisting to switch the patient to the preferred method, giving inaccurate information about unavailable methods, and emphasizing disadvantages of particular methods decreases method uptake and satisfaction.

Currently, there are no, prior contraceptive medical studies that link the use of the preferred method of family planning in the advent of the COVID 19 pandemic. Therefore, a better understanding of factors particular to 
using the preferred method of family planning during the COVID 19 lockdown will enable uptake and satisfactory use of preferred contraceptive methods, slowdown discontinuation rates, and prevent unnecessary method switching (Machiyama et al., 2018; Organization, 2017). In this regard ameliorating the specter of using unpreferred methods of family planning during future lockdowns is paramount in realizing the Sustainable Development Goal (SDG) indicator 3.7.1. This study analyses the prevalence of the preferred methods of contraception during the COVID 19 lockdown in Uganda: A multinomial logistic regression study.

\section{Methods}

\section{Data extraction}

We used data from the post-intervention telehealth survey conducted in 2020 entitled "Using telehealth services to support the continuity of family planning information, access, and utilization during the Covid-19 pandemic in Uganda" for this manuscript. The entire survey covered a sample of 1050 participants of reproductive age (15-60).

\section{Study design}

The post-intervention telehealth survey conducted in 2020 was cross-sectional in design in which participants who met the survey criteria were interviewed using Computer Assisted Telephone Interviews (CATI). The analysis in the survey curtailed both genders between ages (18-60 years) both users and non-users of family planning. The study design included a baseline survey, an intervention, and a post-intervention survey.

\section{Study variables}

The dependent variable for this study, which was the preferred method of family planning, was derived from two specific questions in the post-intervention survey. Question one: Did you use your most preferred method of family planning? This question was defined as a dichotomous variable, differentiating between participants who answered 'Yes' who were coded as ' 1 ' and ' $N o$ ' coded as ' 0 ' Note: after running the logistic regression in model one, participants coded zero were dropped. Question two: Which method of family planning are you using during the COVID 19 lockdown? This question required the participant to choose from the various methods of family planning. Question two was coded into four categories i.e. permanent methods ' 1 ', LARC ' 2 ', SAC ' 3 ', and other methods (traditional methods) ' 4 '. Note: Category 4 was dropped since it included natural and traditional methods.

The independent variables were selected for inclusion in the analysis based on their significance in prior studies on factors that influence the preferred method of family planning. These variables were identified under three categories comprising demographic and socioeconomic factors, intervention factors, and COVID 19 related factors. All variables included in the analysis were carefully selected from the post-intervention survey questionnaire. The independent variables were organized into demographic factors, social-economic, and health-related factors. The demographic and social-economic factors included; age, sex, region, religion, education level, employment status, and marital status. While the health-related factors included: Frequency of Family Planning messages wanted, Using any method of Family Planning, Faced any Challenge, the Biggest challenge faced not to use Family Planning, messages about Family Planning information acquired,

Messages Referral services acquired, Messages about access, Messages number is satisfactory, Messages from the intervention were enough, Most Beneficial Messages, When started using Family Planning, Would recommend using Family Planning, Was able to access/use LARC, More information needed, Would you 
advise to seek Family Planning, Advise partner to use LARC, Stopped using because of COVID, Intervention helped him to start Family Planning, Intervention encouraged him to continue Family Planning, Intervention increased Family Planning knowledge, Prepared to use Family Planning in another lockdown, Would continue using Family Planning even after lockdown, Plan to prevent pregnancy in case of another lockdown, Specific method to use under a lockdown.

\section{Analytical approach}

We analyzed the data at 3 levels of analysis. At level one of the analysis, we employed a univariate analysis which included the rate of prevalence of the most preferred method of family planning used. And a sample description of the demographic, and socioeconomic variables, intervention variables, and COVID 19 related variables. Frequencies and percentages were used to guide the sample description. The second level applied bivariate analysis which focused on establishing the unadjusted relationship between the explanatory variables and the outcome variable - the preferred method of family planning. This approach provided a preliminary analysis of the preferred method of family planning across the individual independent variables. The third level of analysis focused on multivariate analysis to assess the adjusted association of the explanatory variables with unmet need. We used a logistic regression model and the multinomial logistic regression model at the multivariate level to analyze how the factors are associated with the most preferred method of family planning. The binary logistic regression was used because the outcome variables i.e. the preferred method of family planning was dichotomous (did not use the most preferred method as 0 , and used the most preferred method of family planning coded as 1). The multinomial logistic regression was applied because the second categorical dependent variable of the preferred method of family planning had three categories (Using permanent methods coded as 1, Using LARC coded as 2, and Using SAC coded as 3). The dichotomous relationship between the preferred method of family planning and the various independent variables was analyzed using logistic regression. The multinomial regression model was used to examine the association between selected independent variables on permanent methods, LARC and SARC.

\section{Results}

\section{Univariate Analysis}

This study was undertaken using data collected from a telehealth cross-sectional survey conducted in 2020 in Uganda and aimed to analyze the factors responsible for using the preferred method of contraception during the COVID 19 lockdown in Uganda. The results show that factors are widespread among Ugandans with more than $80 \%$ reporting that they used their preferred method of family planning, during the COVID19 lockdown. The study also shows that almost $50 \%$ of the participants used short-acting methods of family planning.

\section{Table 1; Outcome Variable (Binary Logistic Regression)}

\begin{tabular}{|l|c|c|}
\hline Outcome Variable (Binary Logistic Regression) & Frequency & Percent \\
\hline Did not use the Preferred Method of Family Planning & 48 & 19.67 \\
\hline Used the Preferred Method of Family Planning & 196 & 80.33 \\
\hline Total & 244 & 100 \\
\hline
\end{tabular}

Table 2: Outcome Variable (Multinomial Logistic Regression) 


\begin{tabular}{|l|c|c|}
\hline Long Term Acting Reversible Contraceptives & 82 & 34.89 \\
\hline Short Acting Methods & 109 & 46.38 \\
\hline Natural methods & 24 & 10.21 \\
\hline Total & 235 & 100 \\
\hline
\end{tabular}

Table 3: Distribution of respondents by demographic and socio-economic factors

\begin{tabular}{|c|c|c|}
\hline \multirow[t]{2}{*}{ Variable } & Frequency & Percent \\
\hline & $(n=244)$ & \\
\hline \multicolumn{3}{|l|}{ Age of respondent } \\
\hline$<20$ & 15 & 5.3 \\
\hline $20-29$ & 134 & 47.2 \\
\hline $30-39$ & 88 & 31.0 \\
\hline $40+$ & 47 & 16.6 \\
\hline \multicolumn{3}{|l|}{ Sex of the Respondent } \\
\hline Male & 219 & 77.1 \\
\hline Female & 65 & 22.9 \\
\hline \multicolumn{3}{|l|}{ Region of the respondent } \\
\hline Central & 42 & 14.8 \\
\hline Eastern & 48 & 16.9 \\
\hline Western & 31 & 10.9 \\
\hline Northern & 163 & 57.4 \\
\hline \multicolumn{3}{|l|}{ Religion of the respondent } \\
\hline Protestant & 122 & 42.96 \\
\hline Catholic & 105 & 36.97 \\
\hline Moslem & 16 & 5.63 \\
\hline Born again & 33 & 11.62 \\
\hline Others & 8 & 2.82 \\
\hline \multicolumn{3}{|l|}{ Education Level of the Respondent } \\
\hline Less than secondary level & 23 & 8.1 \\
\hline Secondary level & 168 & 59.15 \\
\hline More than secondary & 93 & 32.75 \\
\hline \multicolumn{3}{|l|}{ Employment status of the respondent } \\
\hline No & 81 & 28.52 \\
\hline Yes & 203 & 71.48 \\
\hline \multicolumn{3}{|l|}{ Marital status } \\
\hline Single & 87 & 30.63 \\
\hline Married or in union & 192 & 67.61 \\
\hline Ever been married & 5 & 1.76 \\
\hline
\end{tabular}

Table 4: Distribution of respondents by COVID 19 related factors

\begin{tabular}{|r|c|c|}
\hline Variable & Frequency & Percent \\
\hline Main method of FP used & & 10.29 \\
\hline Permanent & 18 & 39.43 \\
\hline LARC & 69 & 50.29 \\
\hline SARC & 88 & \\
\hline Had Access to FP in the Lockdown & & 92.18 \\
\hline Yes & 224 & \\
\hline
\end{tabular}


Biggest challenge

Failure to get transport to HF

8

Stock out at my health facility Lack of money

Fear of my spouse to know

Fear of unwanted pregnancy (meth expires)

Fear of what happens to my body (expires)

Lack of a health provider to talk to

Would recommend a friend

Yes

Yes

173

2

98.86

1.14

Able to access LARC in lockdown

Yes

120

68.57

31.43

Advise a person to seek FP services

Yes

174

1

0.57

Would advise a partner to use LARC

Yes
149

85.14

No

Stopped using FP coz of lockdown

Yes
28

147

Prepared to use FP in future lockdown

Yes
175 100

0

0

Continue using FP after the lockdown

Yes
No
169

6

Plan to ensure to use FP after lockdown

Use of LARC
Permanent method
Seek partner approval
Others, specify

7

4
48

19

10.86

Best method to use during Lockdown

\begin{tabular}{c|c|c|} 
Yes & 166 & 94.86 \\
\hline No & 9 & 5.14 \\
\hline
\end{tabular}

Table 5: Distribution of respondents by intervention Characteristics

\begin{tabular}{|r|c|c|} 
Variable & Frequency & Percent \\
\hline Messages contained FP information & 2 & 1.14 \\
\hline 1 Not enough & 2 & 0.82 \\
\hline 2 Somehow enough & 4 & 1.65
\end{tabular}




\begin{tabular}{|c|c|c|}
\hline 3 Neutral & 25 & 10.29 \\
\hline 4 Adequate & 81 & 33.33 \\
\hline Extremely adequate & 131 & 53.91 \\
\hline \multicolumn{3}{|l|}{ Messages contained Referral services } \\
\hline 1 Not enough & 21 & 12 \\
\hline 2 Somehow enough & 8 & 4.57 \\
\hline 3 Neutral & 39 & 22.29 \\
\hline 4 Adequate & 45 & 25.71 \\
\hline 5 Extremely adequate & 62 & 35.43 \\
\hline \multicolumn{3}{|l|}{ Messages contained access to FP services } \\
\hline 1 Not enough & 11 & 6.29 \\
\hline 2 Somehow enough & 17 & 9.71 \\
\hline 3 Neutral & 39 & 22.29 \\
\hline 4 Adequate & 46 & 26.29 \\
\hline 5 Extremely adequate & 62 & 35.43 \\
\hline \multicolumn{3}{|l|}{ Satisfied with the intervention } \\
\hline 1 Not enough & 2 & 1.15 \\
\hline 2 Somehow enough & 4 & 2.3 \\
\hline 3 Neutral & 8 & 4.6 \\
\hline 4 Adequate & 50 & 28.74 \\
\hline 5 Extremely adequate & 110 & 63.22 \\
\hline \multicolumn{3}{|l|}{ Number of messages in the intervention } \\
\hline 1 Not enough & 14 & 8 \\
\hline 2 Somehow enough & 7 & 4 \\
\hline 3 Neutral & 13 & 7.43 \\
\hline 4 Adequate & 50 & 28.57 \\
\hline 5 Extremely adequate & 91 & 52 \\
\hline \multicolumn{3}{|l|}{ Best intervention service offered } \\
\hline SMS (information, behavioral and motivation & 68 & 38.86 \\
\hline Counselling & 48 & 27.43 \\
\hline Referral for family planning services & 2 & 1.14 \\
\hline Use of long term reversible contraception & 51 & 29.14 \\
\hline Other, specify & 6 & 3.43 \\
\hline \multicolumn{3}{|l|}{ Point, started using FP } \\
\hline Before the intervention & 182 & 80.53 \\
\hline During the intervention & 30 & 13.27 \\
\hline After the intervention & 14 & 6.19 \\
\hline \multicolumn{3}{|l|}{ What info. Needed to use Modern FP } \\
\hline FP information & 55 & 31.43 \\
\hline Referrals for FP services & 7 & 4 \\
\hline Use of LARC & 63 & 36 \\
\hline Counselling & 41 & 23.43 \\
\hline Other & 9 & 5.14 \\
\hline Intervention supported to start using FP & & \\
\hline
\end{tabular}




\begin{tabular}{|r|c|c|}
\hline Yes & 93 & 53.76 \\
\hline No & 80 & 46.24 \\
\hline Intervention helped to continue using FP & & 95.43 \\
\hline Yes & 167 & 4.57 \\
\hline No & 8 & 98.86 \\
\hline Gained increased knowledge and awareness & & 1.14 \\
\hline
\end{tabular}

Bivariate analysis

Table 6: Percentages of respondents who used the Preferred Method of Family Planning by demographic and Socio Economic Characteristics

\begin{tabular}{|c|c|c|c|}
\hline Variable & $\begin{array}{l}\text { Not using the preferred } \\
\text { Contraceptive method } \\
\text { (\%) }\end{array}$ & $\begin{array}{c}\text { Using preferred } \\
\text { Contraceptive Method } \\
\text { (\%) }\end{array}$ & Total \\
\hline \multicolumn{4}{|l|}{ Age of respondent } \\
\hline$<20$ & 6 & 24 & 30 \\
\hline $20-29$ & 20 & 121 & 141 \\
\hline $30-39$ & 16 & 37 & 53 \\
\hline \multirow[t]{2}{*}{$40+$} & 14 & 14 & 20 \\
\hline & \multicolumn{2}{|l|}{ Pearson $=7.75$} & \\
\hline \multicolumn{4}{|l|}{ Region of the respondent } \\
\hline Central & 16 & 64 & 80 \\
\hline Western & 8 & 18 & 26 \\
\hline Eastern & 4 & 43 & 47 \\
\hline \multirow[t]{2}{*}{ Northern } & 20 & 71 & 91 \\
\hline & Pearson $=6.043$ & $P=0.110$ & \\
\hline \multicolumn{4}{|l|}{ Gender of the respondent } \\
\hline Male & 35 & 153 & 188 \\
\hline \multirow[t]{2}{*}{ Female } & 13 & 43 & 56 \\
\hline & Person $=0.577$ & $p=0.477$ & \\
\hline \multicolumn{4}{|l|}{ Religion of the respondent } \\
\hline Protestant / Anglican & 17 & 62 & 79 \\
\hline Catholic & 20 & 73 & 93 \\
\hline Moslem & 2 & 27 & 29 \\
\hline Seventh Day Adventist & 1 & 5 & 6 \\
\hline \multirow[t]{2}{*}{ Saved/Protestant } & 8 & 29 & 37 \\
\hline & Person $=3.791$ & $p=0.580$ & \\
\hline \multicolumn{4}{|l|}{ Education level } \\
\hline Primary & 7 & 16 & 23 \\
\hline Secondary & 29 & 120 & 149 \\
\hline Tertiary/Vocational & 6 & 42 & 48 \\
\hline \multirow[t]{2}{*}{ University } & 6 & 18 & 24 \\
\hline & Person $=3.684$ & $p=0.298$ & \\
\hline Working Status & & & \\
\hline
\end{tabular}




\begin{tabular}{|c|c|c|c|}
\hline No & 13 & 68 & 81 \\
\hline \multirow[t]{2}{*}{ Yes } & 35 & 128 & 163 \\
\hline & Person $=1.007$ & $p=0.316$ & \\
\hline \multicolumn{4}{|l|}{ Marital Status } \\
\hline Single & 21 & 96 & 117 \\
\hline \multirow[t]{2}{*}{ Married } & 27 & 100 & 127 \\
\hline & Person $=0.423$ & $p=0.516$ & \\
\hline
\end{tabular}

The bivariate distribution of respondents who used the preferred method of family planning according to the selected demographic characteristics are presented in Table 6. The Pearson's Chisquare test reported statistically significant variation in age of the respondent $\left(X^{2}=\mathbf{7 . 7 5} ; \mathbf{p}<\mathbf{0 . 0 5}\right)$. Other characteristics remain insignificant.

Table 7: Percentages of respondents who used the Preferred Method of Family Planning by Intervention related variables

\begin{tabular}{|c|c|c|c|}
\hline Variable & $\begin{array}{l}\text { Not using the preferred } \\
\text { Contraceptive method } \\
\text { (\%) }\end{array}$ & $\begin{array}{c}\text { Using preferred } \\
\text { Contraceptive Method } \\
\text { (\%) }\end{array}$ & Total \\
\hline \multicolumn{4}{|l|}{$\begin{array}{l}\text { FP information received in the } \\
\text { intervention }\end{array}$} \\
\hline Not enough & 1 & 1 & 2 \\
\hline Somehow enough & 2 & 2 & 4 \\
\hline Neutral & 11 & 14 & 25 \\
\hline Adequate & 17 & 64 & 81 \\
\hline \multirow[t]{2}{*}{ Extremely adequate } & 16 & 115 & 131 \\
\hline & \multicolumn{3}{|c|}{ Pearson $=17.766 \quad P=0.001$} \\
\hline \multicolumn{4}{|l|}{ Referral services within messages } \\
\hline Not enough & 9 & 25 & 34 \\
\hline Somehow enough & 4 & 9 & 13 \\
\hline Neutral & 9 & 44 & 53 \\
\hline adequate & 12 & 53 & 65 \\
\hline \multirow[t]{2}{*}{ Extremely adequate } & 13 & 65 & 78 \\
\hline & \multicolumn{3}{|c|}{ Pearson $=2.775 \mathrm{P}=0.596$} \\
\hline \multicolumn{4}{|l|}{$\begin{array}{l}\text { Messages about access to FP } \\
\text { Messages }\end{array}$} \\
\hline Not enough & 8 & 11 & 19 \\
\hline Somehow enough & 4 & 17 & 21 \\
\hline Neutral & 6 & 48 & 54 \\
\hline Adequate & 16 & 54 & 70 \\
\hline \multirow[t]{2}{*}{ Extremely adequate } & 13 & 66 & 79 \\
\hline & \multicolumn{3}{|c|}{ Pearson $=9.6332 \quad P=0.047$} \\
\hline \multicolumn{4}{|l|}{ Satisfied with the intervention } \\
\hline Not enough & 0 & 2 & 2 \\
\hline Somehow enough & 2 & 5 & 7 \\
\hline Neutral & 10 & 10 & 20 \\
\hline Adequate & 8 & 55 & 63 \\
\hline
\end{tabular}




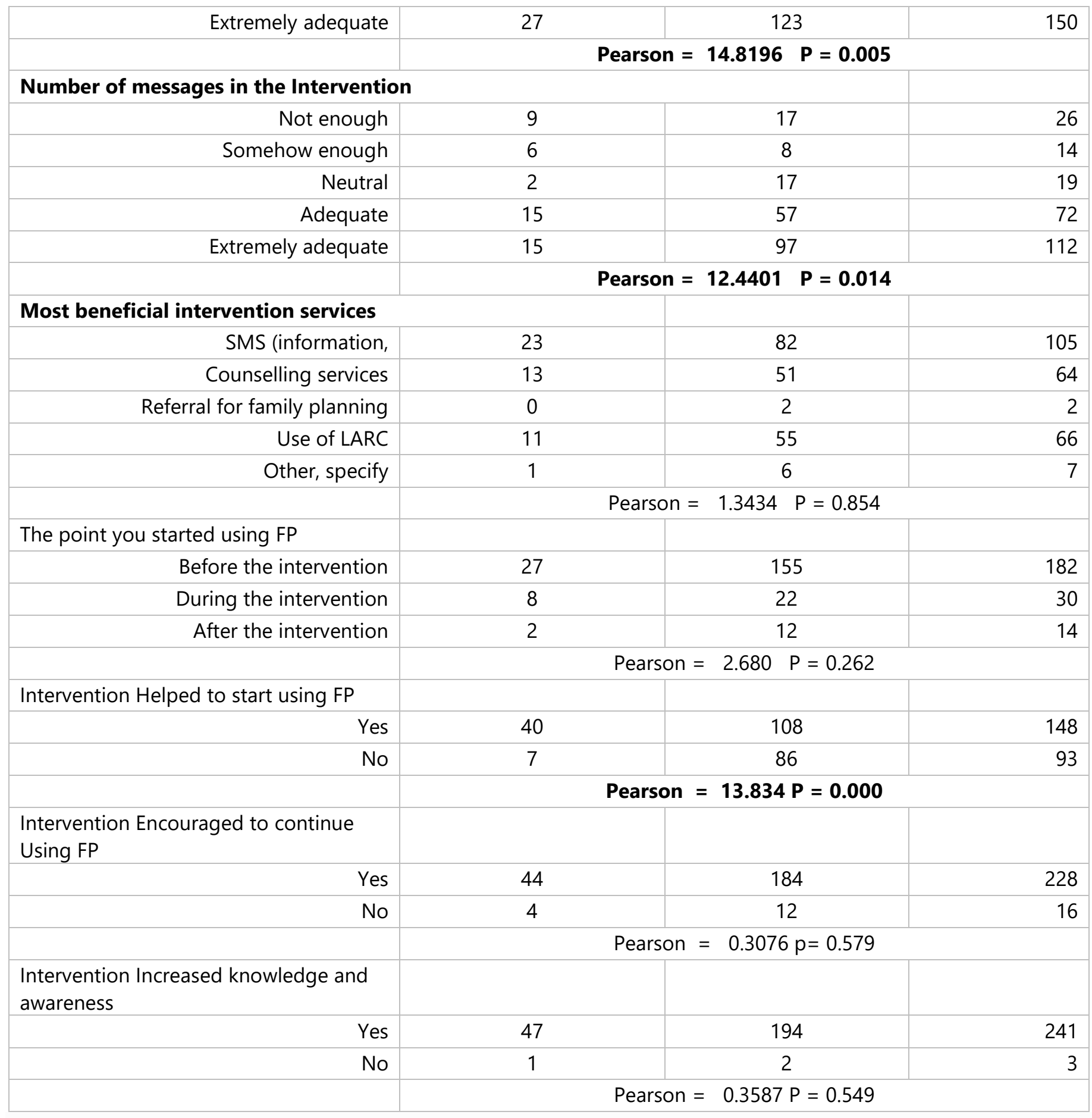

The bivariate distribution of respondents who used the preferred method of family planning according to the selected Intervention related variables is presented in Table 7. The Pearson's Chisquare test reported statistically significant variation in FP information received in the intervention $\left(X^{2}=17.766 ; p<0.001\right)$, Satisfied with the intervention $\left(X^{2}=7.75 ; p<0.05\right)$, Number of messages in the Intervention $\left(X^{2}=14.820 ; p<0.005\right)$, Intervention Helped to start using FP $\left(X^{2}=13.834 ; p<\right.$ 0.000). Other characteristics remain insignificant.

Table 8: Percentages of respondents who used the Preferred Method of Family Planning by COVID 19 related variables

\begin{tabular}{|c|c|c|}
\hline Variable & $\begin{array}{c}\text { Not using the preferred } \\
\text { Contraceptive method (\%) }\end{array}$ & $\begin{array}{c}\text { Using preferred } \\
\text { Contraceptive } \\
\text { Method (\%) }\end{array}$ \\
\hline
\end{tabular}

Total 


\begin{tabular}{|c|c|c|c|}
\hline $\begin{array}{r}\text { Access to FP during COVID } 19 \\
\text { Lockdown }\end{array}$ & & & \\
\hline $\begin{array}{l}\text { Yes } \\
\end{array}$ & 39 & 185 & 224 \\
\hline \multirow[t]{2}{*}{ No } & 9 & 10 & 19 \\
\hline & Pearson $=9.916 \mathrm{P}=0.002$ & & \\
\hline \multicolumn{4}{|l|}{ Currently Using FP } \\
\hline No & 6 & 0 & 6 \\
\hline \multirow[t]{2}{*}{ Yes } & 42 & 196 & 238 \\
\hline & Pearson $=25.117 \mathrm{P}=0.000$ & & \\
\hline \multicolumn{4}{|l|}{ Main Family Planning Method Used } \\
\hline Permanent methods & 2 & 18 & 20 \\
\hline LARC & 13 & 69 & 82 \\
\hline SAC & 21 & 88 & 109 \\
\hline \multirow[t]{2}{*}{ Other FPs } & 5 & 19 & 24 \\
\hline & Pearson $=1.356 \mathrm{P}=0.716$ & & \\
\hline \multicolumn{4}{|l|}{ Biggest challenge during lock down } \\
\hline Failure to get transport & 15 & 86 & 101 \\
\hline Stock out at my Health Facility & 5 & 8 & 13 \\
\hline Lack of money & 1 & 24 & 25 \\
\hline Fear of security personnel & 2 & 1 & 3 \\
\hline Fear to have my spouse & 1 & 4 & 5 \\
\hline Fear of unwanted pregnancy & 2 & 10 & 12 \\
\hline Fear of what happens & 7 & 10 & 36 \\
\hline \multirow[t]{2}{*}{ Lack of a health provider to talk to } & 15 & 34 & 49 \\
\hline & Pearson $=16.250 p=0.023$ & & \\
\hline \multicolumn{4}{|l|}{ Like hood to recommend a friend } \\
\hline $\begin{array}{ll}\text { Yes } \\
\end{array}$ & 43 & 193 & 236 \\
\hline \multirow[t]{2}{*}{ No } & 1 & 3 & 4 \\
\hline & Pearson $=0.1208 \quad \mathrm{P}=0.728$ & & \\
\hline \multicolumn{4}{|l|}{ Ability to use LARC } \\
\hline Yes & 27 & 127 & 154 \\
\hline \multirow[t]{2}{*}{ No } & 17 & 69 & 86 \\
\hline & Pearson $=0.1841 p=0.668$ & & \\
\hline \multicolumn{4}{|l|}{ More information need } \\
\hline FP information & 17 & 64 & 81 \\
\hline Referrals for FP services & 2 & 9 & 11 \\
\hline Use of LARC & 17 & 68 & 85 \\
\hline Counselling & 9 & 46 & 55 \\
\hline \multirow[t]{2}{*}{ Other } & 3 & 9 & 12 \\
\hline & Pearson $=0.707 \quad \mathrm{P}=0.951$ & & \\
\hline \multicolumn{4}{|l|}{ Would advise FP use } \\
\hline Yes & 47 & 195 & 242 \\
\hline \multirow[t]{2}{*}{ No } & 1 & 1 & 2 \\
\hline & Pearson $=1.174 \mathrm{P}=0.279$ & & \\
\hline \multicolumn{4}{|l|}{ Advise the partner to use LARC } \\
\hline $\begin{array}{ll}\text { Yes } \\
\end{array}$ & 41 & 165 & 206 \\
\hline \multirow[t]{2}{*}{ No } & 7 & 30 & 37 \\
\hline & Pearson $=0.0192 \mathrm{P}=0.890$ & & \\
\hline $\begin{array}{r}\text { Stopped using FP because of } \\
\text { Lockdown }\end{array}$ & & & \\
\hline
\end{tabular}




\begin{tabular}{|c|c|c|c|}
\hline Yes & 8 & 29 & 37 \\
\hline No & 36 & 166 & 202 \\
\hline & Pearson $=0.301 \mathrm{P}=0.584$ & & \\
\hline \multicolumn{4}{|l|}{$\begin{array}{r}\begin{array}{r}\text { Preparation to use FP in another } \\
\text { lockdown }\end{array}\end{array}$} \\
\hline Yes & 43 & 196 & 239 \\
\hline No & 5 & 0 & 5 \\
\hline & \multicolumn{2}{|l|}{ Pearson $=20.844 p=0.000$} & \\
\hline \multicolumn{4}{|l|}{$\begin{array}{r}\text { Willingness to continue using FP after } \\
\text { a lockdown }\end{array}$} \\
\hline Yes & 40 & 190 & 230 \\
\hline \multirow[t]{2}{*}{ No } & 8 & 6 & 14 \\
\hline & Pearson $=13.196 p=0.000$ & & \\
\hline \multicolumn{4}{|l|}{$\begin{array}{r}\text { Plan to ensure to use FP in another } \\
\text { Lockdown }\end{array}$} \\
\hline Use of LARC & 33 & 115 & 148 \\
\hline Permanent method & 2 & 7 & 9 \\
\hline Seek partner approval & 7 & 54 & 61 \\
\hline \multirow[t]{2}{*}{ Others, specify } & 5 & 20 & 25 \\
\hline & Pearson $=3.303 p=0.347$ & & \\
\hline \multicolumn{4}{|l|}{$\begin{array}{r}\text { Best preferred method to use under a } \\
\text { lockdown }\end{array}$} \\
\hline Yes & 45 & 187 & 232 \\
\hline No & 3 & 9 & 12 \\
\hline & Pearson $=0.227 p=0634$ & & \\
\hline
\end{tabular}

The bivariate distribution of respondents who used the preferred method of family planning according to the selected COVID 19 related variables are presented in Table 8. The Pearson's Chisquare test reported statistically significant variation in On access to FP during COVID 19. $\left(X^{2}=\right.$ 9.916; $P=0.002)$, participants currently using FP during the lockdown $\left(X^{2}=25.117 ; p<0.000\right)$, Challenges during the lockdown $\left(X^{2}=16.250 ; p<0.023\right)$, those who were prepared to use FP in another lockdown $\left(X^{2}=20.844 ; p<0.000\right)$, Willingness to continue using FP after the lockdown $\left(X^{2}\right.$ $=13.196 ; p<0.000)$. Other characteristics remain insignificant.

\section{Multinomial regression results}


Table 9: Predictors of using the Most Preferred method of Family Planning during the COVID 19 Lockdown

\begin{tabular}{|c|c|c|c|c|c|c|c|c|c|c|c|}
\hline \multirow[b]{3}{*}{ Independent Variables } & \multirow{2}{*}{\multicolumn{3}{|c|}{$\begin{array}{l}\text { Model I [Logistic] } \\
\text { Participants using the most } \\
\text { preferred Method FP }(\mathrm{n}=\mathbf{2 3 9})\end{array}$}} & \multirow{2}{*}{\multicolumn{4}{|c|}{$\begin{array}{l}\text { Model II [Multinomial] } \\
\text { Using Permanent Methods of FP }(n= \\
\text { 172) }\end{array}$}} & \multirow{2}{*}{\multicolumn{4}{|c|}{$\begin{array}{l}\text { LARC (Long Acting Reversible Methods) ( } n= \\
\text { 172) }\end{array}$}} \\
\hline & & & & & & & & & & & \\
\hline & Odds Ratio & Std. Err. & p-value & RRR & Std.Err & p-value & $95 \% \mathrm{Cl}$ & RRR & Std.Err & p-value & $95 \% \mathrm{Cl}$ \\
\hline \multicolumn{12}{|l|}{ Age of the Respondent } \\
\hline$<19$ Years & 1 & & & 1 & & & & 1 & & & \\
\hline 20-29 Years & 0.721 & 0.522 & 0.650 & 1.195 & 1.276 & 0.867 & 0.148 & 0.486 & 0.332 & 0.290 & 0.128 \\
\hline 30-39 Years & 0.178 & 0.140 & 0.03 & 0.604 & 0.778 & 0.696 & 0.048 & 0.515 & 0.397 & 0.389 & 0.114 \\
\hline $40>$ Years & 0.127 & 0.118 & 0.03 & 3.794 & 6.413 & 0.430 & 0.138 & 1.199 & 1.261 & 0.863 & 0.152 \\
\hline \multicolumn{12}{|l|}{ Had Access to FP } \\
\hline Yes & 1 & & & 1.000 & & & & 1 & & & \\
\hline No & 0.488 & 0.325 & 0.280 & 1.370 & 0.000 & 0.995 & 0.000 & 0.192 & 0.245 & 0.196 & 0.016 \\
\hline \multicolumn{12}{|l|}{ Biggest Challenge } \\
\hline Failure to get transport to $\mathrm{HF}$ & 1 & & & 1 & & & & 1 & & & \\
\hline Stock out at my health facility & 0.506 & 0.438 & 0.430 & 1.120 & 0.000 & 0.994 & 0.000 & & & 0.840 & 0.194 \\
\hline Fear of security personnel & 0.019 & 0.030 & 0.01 & & & & & & & & \\
\hline Fear of my spouse & 0.075 & 0.101 & 0.05 & 3.130 & 0.000 & 0.996 & 0.000 & 9.910 & 0.000 & 0.994 & 0.000 \\
\hline Fear of unwanted pregnancy & 0.279 & 0.283 & 0.210 & 2.085 & 3.223 & 0.635 & 0.101 & 14.490 & 16.739 & 0.021 & 1.506 \\
\hline Fear if implant expires & 0.665 & 0.447 & 0.540 & 0.465 & 0.425 & 0.402 & 0.077 & 3.943 & 2.122 & 0.011 & 1.374 \\
\hline Lack of health provider & 0.730 & 0.399 & 0.570 & 0.354 & 0.433 & 0.396 & 0.032 & 1.672 & 0.880 & 0.329 & 0.596 \\
\hline \multicolumn{12}{|l|}{ Access to FP services (Msgs) } \\
\hline 1 Not enough & 1 & & & 1 & & & & 1 & & & \\
\hline 2 Somehow enough & 4.676 & 4.648 & 0.120 & 0.628 & 1.147 & 0.799 & 0.018 & 0.703 & 0.741 & 0.738 & 0.089 \\
\hline 3 Neutral & 3.766 & 3.140 & 0.110 & 1.181 & 1.801 & 0.913 & 0.060 & 1.334 & 1.304 & 0.768 & 0.196 \\
\hline 4 Adequate & 2.615 & 1.987 & 0.210 & 0.847 & 1.226 & 0.909 & 0.050 & 0.717 & 0.666 & 0.720 & 0.116 \\
\hline 5 Extremely adequate & 1.858 & 1.440 & 0.420 & 0.259 & 0.375 & 0.351 & 0.015 & 1.230 & 1.128 & 0.822 & 0.204 \\
\hline \multicolumn{12}{|l|}{ Number of Msgs } \\
\hline 1 Not enough & 1 & & & 1 & & & & 1 & & & \\
\hline 2 Somehow enough & 0.508 & 0.441 & 0.435 & 4.340 & 14.833 & 1.000 & 0.000 & 3.602 & 4.665 & 0.322 & 0.285 \\
\hline 5 Extremely adequate & 6.377 & 4.305 & 0.006 & 1.860 & 3.530 & 0.993 & 0.000 & 4.899 & 4.087 & 0.057 & 0.955 \\
\hline Intervention Helped you to start & & & & & & & & & & & \\
\hline
\end{tabular}




\begin{tabular}{|c|c|c|c|c|c|c|c|c|c|c|c|}
\hline Yes & 1 & & & 1 & & & & 1 & & & \\
\hline No & 7.569 & 5.216 & 0.003 & 0.971 & 0.634 & 0.964 & 0.270 & 0.413 & 0.178 & 0.040 & 0.178 \\
\hline \multicolumn{12}{|l|}{ Continue using FP after the lockdown } \\
\hline Yes & 1 & & & 1 & & & & 1 & & & \\
\hline No & 0.016 & 0.015 & 0.000 & 1.560 & 0.000 & 0.996 & 0.000 & 1.497 & 1.717 & 0.725 & 0.158 \\
\hline _cons & 23.828 & 38.839 & 0.052 & 2.630 & 1.130 & 0.997 & 0.000 & 2.957 & 6.593 & 0.627 & 0.037 \\
\hline \multirow[t]{4}{*}{ Note:_cons estimates baseline odds. } & & & & SARC & \multicolumn{5}{|c|}{ (base outcome) } & & \\
\hline & & & & & & & & & & & \\
\hline & & & & \multirow{2}{*}{\multicolumn{8}{|c|}{\begin{tabular}{l|l} 
Note:_cons estimates baseline relative risk for each outcome. \\
Note: 4 observations completely determined. Standard errors questionable.
\end{tabular}}} \\
\hline & & & & & & & & & & & \\
\hline
\end{tabular}

\section{Model I [LOGISTIC REGRESSION]}

Table 9 presents the logistic regression (Model I) estimates of odds ratio and the multinomial regression (Model II) estimates of relative risk ratios (RRR) for selected variables of participants who used any of the three types of the most preferred modern method of family planning i.e. (permanent methods, LARC and SARC). Model I reveals that for using the most preferred method of family planning, the study found that the age of the sexual partner significantly predicted the use of the preferred method of family planning among sexually active partners. Sexual partners aged 30-39 years were less likely $(\mathrm{OR}=0.2 ; \mathrm{Cl}$ 0.04-0.83) to use the most preferred method of family planning compared to those less than 20 years of age. More so, sexual partners aged 40 years and above were less likely $(\mathrm{OR}=0.1 ; \mathrm{Cl} 02-0.78)$ to use the most preferred method of family planning compared to those of less than 20 years of age. Participants whose biggest challenge to family planning information, access, and utilization was lack of money to access family planning methods were more likely (OR = 22.0; $\mathrm{Cl}$ 1.93-258.53) to use the preferred method of family planning while those whose biggest challenges were Fear to have their spouse know that they are using family planning and fear of security personnel/lack of pass to go to the health facility were less likely $(\mathrm{OR}=0.01 ; \mathrm{Cl} 0.00-0.44$ and, $\mathrm{OR}=0.05 ; \mathrm{Cl} 0.01$ 1.04 ) to use their most preferred method of Family planning compared to those whose biggest challenge was Failure to get transport to the health facility. In the post-intervention survey, participants who subscribed to receive the maximum number of messages during the intervention period are more likely (OR $=6.38 ; \mathrm{Cl} 1.70-23.94)$ to use their most preferred method of Family planning compared to those who did not receive enough messages. More so, the participants whom the intervention helped to start using family planning for the first time were more likely $(\mathrm{OR}=7.57 ; \mathrm{Cl} 1.96-29.22)$ to use their most preferred method of Family planning compared to those who were already using family planning. For participants who had no willingness to continue using family planning even after the lockdown is over were less likely $(\mathrm{OR}=0.02 ; \mathrm{Cl} 0.00-0.10)$ to use the preferred method of Family planning compared to those who were not willing to continue using family planning. 


\section{Model II [MULTINOMIAL LOGISTIC REGRESSION]}

In Model II participants who had fear for unwanted pregnancy during the COVID 19 lockdown were relatively more likely ( $R R R=14.49 ; \mathrm{Cl} 1.51-139.43$ ) to use LARC as a preferred method of family planning compared to those who used SARC as their most preferred method of family planning. Participants who feared that implant might expire in their bodies during the COVID 19 lockdown were relatively more likely $(R R R=3.94 ; C l 1.73$ 11.32) to use LARC as a preferred method of family planning compared to those who used SARC as their most preferred method of family planning. Participants whom the intervention helped to start using during the COVID 19 lockdown were relatively less likely ( $R R R=0.41 \mathrm{CI} 0.18-0.96)$ to use $L A R C$ as a preferred method of family planning compared to those who used SARC as their most preferred method of family planning. However, there were no significant differences observed in age categories, access to FP services and the number of messages received during the intervention, and those with the willingness to use family planning even after the lockdown.

\section{Discussion}

This study assessed the associations between selected explanatory variables and unmet need in Ghana. The patterns of high unmet need as reported in unmet need prevalent country like ours (35.17\%) reveals that unmet need increased among Pentecostal Protestants, women who had more than one marriage unions, women who did not know the couple's desired number of children, and women who had an additional number of living biological child. Unmet need decreased among women as they grew older, among those employed and women with an additional number of the ideal child. Unmet need for spacing was positive among women who did not know the couple's desired number of children, as the age of women getting married increases and women who had an additional living biological child. Unmet need for spacing decreased as women grew older, among rural women and women employed. Unmet need for limiting was positive among women who had at least secondary education, women from wealthier households, and among women with an additional living biological child. Unmet need for limiting decreased as women had an additional ideal child.

The study assessed the associations between the selected independent variables and the preferred method of family planning. The study found that the age of the sexual partner significantly predicted the use of the preferred method of family planning among sexually active partners. The odds of using the most preferred method among sexual partners aged 30-39 were 0.2 times, higher compared to those of less than 20 years of age. More so, the odds of using the most preferred method among sexual partners aged $40+$ years were 0.1 times, higher compared to those of less than 20 years of age. These findings could be explained by the desire for child spacing, some of these couples have already had the desired number of children and are informed of Family Planning services.

The study also found that challenges to access family planning services during the COVID 19 lockdown significantly predicted the use of the preferred method of family planning among sexually active partners.

The odds of using the most preferred method among sexual partners who lacked money as their biggest challenge to access family planning during the COVID 19 lockdown were 22.3 times, higher compared to those whose biggest challenge was the failure to get transport to access health facilities during the COVID 19 lockdown in Uganda (Bolarinwa, 2021; Chandrasekaran et al., 2020)

The odds of using the most preferred method among sexual partners who Feared to have their spouse know 
that they are using family planning as their biggest challenge to access family planning during the COVID 19 lockdown were 0.1 times, higher compared to those whose biggest challenge was the failure to get transport to access health facilities during the COVID 19 lockdown in Uganda. More so the odds of using the most preferred method among sexual partners who feared to have their spouse know that she is using family planning as their biggest challenge to access family planning during the COVID 19 lockdown were 0.1 times, higher compared to those whose biggest challenge was the failure to get transport to access health facilities during the COVID 19 lockdown in Uganda (Kibira et al., 2020).

The study found that telehealth intervention to helping sexually active partners to start using family planning significantly predicted the use of the preferred method of family planning among sexually active partners. The odds of using the most preferred method among those whom the telehealth intervention helped to start using family planning were 7.6 times, higher compared to those whom the intervention did not help to start using Family planning.

The study found that willingness to use family planning even after the lockdown significantly predicted the use of the preferred method of family planning among sexually active partners. The odds of using the most preferred method among sexual partners who are willing to use family planning even after the lockdown were 0.01 times, higher compared to those who had no willingness to use family planning after the COVID 19 lockdown.

\section{Multinomial}

For participants who had no willingness to continue using family planning even after the lockdown is over, were less likely $(\mathrm{OR}=0.02 ; \mathrm{Cl} 0.00-0.10)$ to use the preferred method of Family planning compared to those who were not willing to continue using family planning. This is better explained by the theory of planned behavior which is used to predict and understand behaviors. It posits that behaviors are immediately determined by behavioral intentions, which in turn are determined by a combination of three factors: attitude toward the behavior, subjective norms, and perceived behavioral control (Agus et al., 2019; Ajzen, 1985).

Our study detected that participants who had fear for unwanted pregnancy were relatively more likely to use Long term acting Reversible contraceptives than Short Term Acting reversible contraceptives as their preferred method of family planning during the COVID 19 lockdown. This finding is consistent with other studies elsewhere that confirm that Long-acting reversible contraception, or LARC, methods provide reliable, long-term, highly effective prevention of pregnancy after one-time placement of a device. In times of a pandemic like the COVID 19 Lockdown, such methods are preferable for longer use, sometimes termed as "forgettable" (Curtis \& Peipert, 2017; Parks \& Peipert, 2016).

In addition to the finding, the Multinomial logistic regression found that participants who feared that implant 
might expire in their bodies during the COVID 19 lockdown were relatively more likely to use LARC as a preferred method of family planning compared to those who use SARC. This means that participants who would easily forget that they have an implant that is about to expire are believed to have low adherence to SARC. Studies elsewhere are in agreement that, family planning users are skeptical of using methods that have high levels of adherence such as taking a pill daily, receiving an injection every 3 months, or placing a ring or patch. This finding builds on research that identified challenges of using SARC and predicted nonperfect adherence. The safety, efficacy, and long-term cost-benefit factors of long-acting reversible contraception (LARC) use in the adolescent population have been well established (Birgisson et al., 2015; Cea Soriano et al., 2015; Kipps, 2015).

Lastly, Participants whom the intervention helped to start using family planning during the COVID 19 lockdown were relatively less likely to use LARC as a preferred method of family planning compared to those who used SAC. The reason might be that first-time family planning users are less likely to choose a long-term method, simply because they are weighing options beyond using LARC against SAC. This finding was as well consistent with other studies, for first-time users of contraceptives who choose Short-term Acting Reversible Contraceptives (SARC) over LARC (Bostick et al., 2020; Machado et al., 2020; Tekle, 2020).

\section{Conclusion}

The results show that increasing age, lack of money as the biggest challenge, comes with using the preferred methods of family planning during the lockdown. It also showed that participants who had fear for unwanted pregnancy and fear that an implant might get expired inside a woman's body were more likely to use Long Term Acting Reversible contraceptives. Last but not least, interventions on family planning information, access, and utilization are beneficial for first-time starters of contraceptive use but many people will continuously use.

\section{Availability of data and materials}

The anonymized datasets used and/or analyzed during the current study are available from the corresponding author on reasonable request.

\section{Abbreviations}

Cl: Confidence interval

FP: Family planning

LMICs: Low and middle income countries

OR: $\quad$ Odds ratio

RRR: Relative risk ratio

SSA: Sub-Saharan Africa

LARC: Long Acting Reversible Contraceptives

SAC: Short Acting Contraceptives 


\section{References}

Agus, Y., Pamungkasari, E. P., \& Soemanto, R. B. (2019). Theory of planned behavior: determinants of the use of modern family planning method. Journal of Maternal and Child Health, 4(5), 369-379.

Ahinkorah, B. O., Ameyaw, E. K., Seidu, A.-A., Agbaglo, E., Budu, E., Mensah, F., Adu, C., \& Yaya, S. (2020). Sexual violence and unmet need for contraception among married and cohabiting women in sub-Saharan Africa: Evidence from demographic and health surveys. Plos One, 15(11), e0240556.

Ahmed, S., Li, Q., Liu, L., \& Tsui, A. O. (2012). Maternal deaths averted by contraceptive use: an analysis of 172 countries. The Lancet, 380(9837), 111-125.

Ajzen, I. (1985). From intentions to actions: A theory of planned behavior. In Action control (pp. 11-39). Springer.

Birgisson, N. E., Zhao, Q., Secura, G. M., Madden, T., \& Peipert, J. F. (2015). Preventing unintended pregnancy: the contraceptive CHOICE project in review. Journal of Women's Health, 24(5), 349-353.

Bitzer, J., Gemzell-Danielsson, K., Roumen, F., Marintcheva-Petrova, M., van Bakel, B., \& Oddens, B. J. (2012). The CHOICE study: effect of counselling on the selection of combined hormonal contraceptive methods in 11 countries. The European Journal of Contraception \& Reproductive Health Care, 17(1), 65-78.

Bolarinwa, O. A. (2021). Factors associated with limited access to condoms and sources of condoms during the COVID-19 pandemic in South Africa. MedRxiv, 2009-2020.

Bostick, E. A., Greenberg, K. B., Fagnano, M., Baldwin, C. D., Halterman, J. S., \& Yussman, S. M. (2020). Adolescent Self-Reported Use of Highly Effective Contraception: Does Provider Counseling Matter? Journal of Pediatric and Adolescent Gynecology, 33(5), 529-535.

Cea Soriano, L., Wallander, M., Andersson, S., Filonenko, A., \& García Rodríguez, L. A. (2015). The continuation rates of long-acting reversible contraceptives in UK general practice using data from The Health Improvement Network. Pharmacoepidemiology and Drug Safety, 24(1), 52-58.

Chandrasekaran, S., Diamond-Smith, N., Srinivasan, K., \& Dalvie, S. (2020). Preparing for an Increased Need for Abortion Access in India during and after COVID-19: Challenges and Strategies. Studies in Family Planning, 51(4), 377-383.

Chigbu, B., Onwere, S., Aluka, C., Kamanu, C., Okoro, O., \& Feyi-Waboso, P. J. C. (2010). Contraceptive choices of women in rural Southeastern Nigeria. Nigerian Journal of Clinical Practice, 13(2).

Cleland, J., Bernstein, S., Ezeh, A., Faundes, A., Glasier, A., \& Innis, J. (2006). Family planning: the unfinished agenda. Lancet, 368(9549), 1810-1827. https://doi.org/10.1016/S0140-6736(06)69480-4

Curtis, K. M., \& Peipert, J. F. (2017). Long-acting reversible contraception. New England Journal of Medicine, 376(5), 461-468.

Dasgupta, A., Kantorová, V., \& Ueffing, P. (2020). The impact of the COVID-19 crisis on meeting needs for family planning: a global scenario by contraceptive methods used. Gates Open Research, 4.

Do, M., \& Kurimoto, N. (2012). Women's empowerment and choice of contraceptive methods in selected African countries. International Perspectives on Sexual and Reproductive Health, 23-33.

Ganatra, B., \& Faundes, A. (2016). Role of birth spacing, family planning services, safe abortion services and post-abortion care in reducing maternal mortality. Best Practice \& Research Clinical Obstetrics \& Gynaecology, 36, 145-155.

Kibira, S. P. S., Karp, C., Wood, S. N., Desta, S., Galadanci, H., Makumbi, F. E., Omoluabi, E., Shiferaw, S., Seme, A., \& Tsui, A. (2020). Covert use of contraception in three sub-Saharan African countries: a qualitative exploration of motivations and challenges. BMC Public Health, 20, 1-10.

Kipps, S. (2015). Fitting and removing long-acting reversible contraception. Nurse Prescribing, 13(10), 498503.

Machado, R. B., Ushikusa, T. E., Monteiro, I. M. U., Guazzelli, C. A. F., Bella, Z. J. di, Politano, C. A., \& Sakamoto, L. C. (2020). Different Perceptions among Women and Their Physicians Regarding Contraceptive Counseling: Results from the TANCO Survey in Brazil. Revista Brasileira de Ginecologia e Obstetrícia, 42(5), 255-265.

Machiyama, K., Obare, F., Chandra-Mouli, V., Chou, D., Festin, M., Khosla, R., Kiarie, J., Say, L., \& Thatte, N. (2018). Accelerating uptake of voluntary, rights-based family planning in developing countries.

Organization, W. H. (2017). Family planning evidence brief: accelerating uptake of voluntary, rights-based 
family planning in developing countries.

Parks, C., \& Peipert, J. F. (2016). Eliminating health disparities in unintended pregnancy with long-acting reversible contraception (LARC). American Journal of Obstetrics and Gynecology, 214(6), 681-688.

Riley, T., Sully, E., Ahmed, Z., \& Biddlecom, A. (2020). Estimates of the potential impact of the COVID-19 pandemic on sexual and reproductive health in low-and middle-income countries. International Perspectives on Sexual and Reproductive Health, 46, 73-76.

Roberton, T., Carter, E. D., Chou, V. B., Stegmuller, A. R., Jackson, B. D., Tam, Y., Sawadogo-Lewis, T., \& Walker, N. (2020). Early estimates of the indirect effects of the COVID-19 pandemic on maternal and child mortality in low-income and middle-income countries: a modelling study. The Lancet Global Health, 8(7), e901-e908.

Sharma, K. A., Zangmo, R., Kumari, A., Roy, K. K., \& Bharti, J. (2020). Family planning and abortion services in COVID-19 pandemic. Taiwanese Journal of Obstetrics and Gynecology.

Tekle, M. G. (2020). CONTRACEPTIVE METHOD MIX AMONG REPRODUCTIVE AGE WOMEN IN FOUR EMERGING REGIONS OF ETHIOPIA; MULTINOMIAL LOGISTIC REGRESSION. 31st EPHA Annual Conference.

Tewabe, T., Ayalew, T., Abdanur, A., Jenbere, D., Ayehu, M., Talema, G., \& Asmare, E. (2020). Contraceptive use and associated factors among sexually active reproductive age HIV positive women attending ART clinic at Felege Hiwot Referral Hospital, Northwest Ethiopia: A cross-sectional study. Heliyon, 6(12), e05653. https://doi.org/https://doi.org/10.1016/j.heliyon.2020.e05653

\section{Acknowledgements}

We would like to thank the participants that contributed to this study.

\section{Funding}

The project was funded by the Makerere University Research and Innovation Fund. The views expressed in this publication are those of the author(s) and not necessarily those of the Makerere University.

\section{Author information}

Affiliations

\section{Population Studies, Makerere University, Kampala, Uganda}

Julius Sseninde, Allen Kabagenyi, Ronald Wasswa

\section{National Population Council, Kampala, Uganda,}

Betty Kyaddondo

\section{Community health, Makerere University School of Public Health, Kampala, Uganda,}

Evelyne Nyacho

\section{Institute of Infectious diseases, Research Unit, Makerere University, Kampala, Uganda}




\section{Contributions}

$J S$ and $\mathrm{AK}^{1}$ conceptualized the study. RW contributed to data management, EN translation and transcription. JS led data analysis. Data were coded by JS with coding decisions discussed with RW, $E N$ and $A K$ reviewed the manuscript. AK provided supervisory support and BK together with $A K^{2}$ oversaw the study. The final manuscript was approved by all authors. All authors read and approved the final manuscript.

\section{Corresponding author}

Correspondence to Julius Sseninde

\section{Ethics declarations}

Ethics approval and consent to participate

Ethical approval was obtained from the Mild May Research Ethic Committee (MUREC) Uganda. Informed written consent was obtained from all participants in interviews and focus group.

\section{Consent for publication}

All included data are anonymised.

\section{Competing interests}

None declared.

\section{Additional information}

Publisher's Note

Springer Nature remains neutral with regard to jurisdictional claims in published maps and institutional affiliations. 
